\title{
CAPIM-GORDURA (Melinis minutiflora P. Beauv.), UMA GRAMÍNEA EXÓTICA QUE COMPROMETE A RECUPERAÇÃO DE ÁREAS DEGRADADAS EM UNIDADES DE CONSERVAÇÃ ${ }^{1}$
}

\author{
Carlos Romero Martins ${ }^{2}$, Laércio Leonel Leite ${ }^{3}$ e Mundayatan Haridasan ${ }^{4}$
}

\begin{abstract}
RESUMO - O capim-gordura, Melinis minutiflora, é uma gramínea de origem africana que invade áreas degradadas da região do Cerrado em detrimento das espécies nativas. A interferência do capim-gordura no processo de sucessão e o efeito da queimada controlada sobre essa gramínea foram estudados em uma cascalheira localizada no Parque Nacional de Brasília. O estudo foi realizado numa área experimental em que, em 1993, havia sido implantado um experimento para avaliar o estabelecimento de gramíneas nativas pela técnica do "coquetel de sementes". Mesmo não estando entre as espécies semeadas, o capim-gordura estabeleceu-se nas parcelas experimentais, onde se incorporou torta de mamona ou turfa. Metade das parcelas que apresentaram os melhores índices de cobertura do solo foi queimada no final da estação seca, em setembro de 1998. Em maio de 1999, oito meses após a queimada verificou-se redução da cobertura do substrato superior a 50\%, em comparação com a cobertura antes da queimada. Nos anos subseqüentes, o índice de cobertura do substrato voltou a crescer, atingindo, em maio de 2001, os valores observados antes da queimada. Durante esse período, notou-se, nas parcelas queimadas e nãoqueimadas, aumento na contribuição do capim-gordura para o índice total de cobertura do solo, em detrimento das espécies nativas. O uso do fogo não foi eficaz no controle dessa invasora. Assim, em programas de recuperação de áreas degradadas em áreas protegidas é necessário controlar as espécies invasoras exóticas para permitir o estabelecimento de um maior número de espécies nativas do Cerrado.
\end{abstract}

Palavras-chave: Áreas protegidas, ecossistemas, gestão ambiental, cerrado.

\section{MOLASSES GRASS (Melinis minutiflora P. Beauv.), AN EXOTIC SPECIES COMPROMISING THE RECUPERATION OF DEGRADED AREAS IN CONSERVATION UNITS}

\begin{abstract}
Melinis minutiflora is an African grass that invades degraded areas in the cerrado endangering native species. The effects of this invasion and of burning on Melinis minutiflora propagation were investigated in a gravel-mining pit, within the National Park of Brasilia. This study was conducted in an area where an experimental field was set up in 1993 to study the establishment of native grasses after sowing with seed mixture. Melinis minutiflora established itself in all experimental plots where castor oil cake or peat was incorporated as amendments before sowing, even though its seeds were not present in the mixture. Half of the experimental plots, which showed the highest indices of vegetation cover in May 1998, were burnt at the end of the dry season in September 1998. Eight months later, May 1999, a reduction of more than 50\% in the vegetation cover of the burnt plots was recorded compared to the vegetative cover before the fire. During the subsequent years, the vegetation cover increased until it reached the pre-fire levels by May 2001.
\end{abstract}

\footnotetext{
${ }^{1}$ Recebido para publicação em 16.6.2003 e aceito para publicação em 10.8.2004.

${ }^{2}$ IBAMA/DILIC/COGEL, Avenida L-4 Norte SAIN. 70800-200 Brasília, DF. E.mail: <carlos.martins@ibama.gov.br>.

${ }^{3}$ Universidade Católica de Brasília, SGAN 916, módulo B. 70790-160 Brasília, DF.

${ }^{4}$ Departamento de Ecologia da Universidade de Brasília. 70.919-970 Brasília, DF.
} 
During this period, the contribution of Melinis minutiflora increased gradually to the detriment of native species, showing that burning was not effective in controlling the invasion of this alien species. Thus, it seems essential to control the invasion of Melinis minutiflora to promote the establishment of native species during recuperation of degraded patches in protected areas such as national parks.

Key words for indexing: Protected areas, ecosystems, environmental management, savannas.

\section{INTRODUÇÃO}

O Brasil possui uma das maiores biodiversidades mundiais, e grande parte dela encontra-se no bioma cerrado. O cerrado abrange 200 milhões de hectares (22\% do território nacional), compreendendo uma larga variedade de fisionomias savânicas que dominam o Ccentro-Oeste brasileiro. Matas de galerias desenvolvemse ao longo dos rios e córregos e matas secas medram sobre solos ricos, bem como vegetação de transição nas bordas com outros biomas. Sua flora é rica e possui 6671 taxa nativos, distribuídos em 170 famílias e 1.144 gêneros (MENDONÇA et al. 1998, UNESCO, 2001).

A invasão de espécies exóticas representa um grave problema para o funcionamento dos ecossistemas e ameaça a diversidade vegetal nas unidades de conservação. No Brasil, os estudos sobre invasão biológica em áreas protegidas são escassos, provavelmente porque o impacto é relativamente lento, no longo prazo, e pouco evidente nas fases de estabelecimento e dispersão (D'ANTONIO VITOUSEK, 1992; KLINK, 1994; MOROSONIe KLINK, 1997; FREITAS, 1999; MARTINS et al. 2001; SILVA, 2003). Gramíneas de origem africana (Brachiaria decumbens Stapf, Andropogon gayanus Kunth, Hyparrhenia rufa Stapf, Melinis minutiflora) foram introduzidas no Brasil, acidentalmente ou para fins comerciais, e se espalharam por grandes extensões de ecossistemas naturais, deslocando espécies nativas graças à sua agressividade e ao seu grande poder competitivo (BARUCH et al., 1985; PIVELLO et al., 1999). Hoje, essas espécies encontram-se bastante disseminadas no Brasil. No Distrito Federal, segundo Filgueiras (1991), já foram identificadas 305 espécies de gramíneas, e as espécies exóticas representam cerca de $20 \%$ do total.

Melinis minutiflora (capim-gordura) é uma gramínea de origem africana, perene, $\mathrm{C}_{4}$, reproduz-se tanto por semente como vegetativamente e foi introduzida em muitos países tropicais como forrageira, podendo ser considerada atualmente como uma espécie naturalizada. Essa gramínea é sensível ao fogo e está adaptada à condição de baixa fertilidade de solo. No entanto, em estudos realizados em casa de vegetação, tendo como substrato Latossolo Vermelho-Amarelo, o crescimento inicial do capim-gordura foi favorecido pela aplicação de $\mathrm{P}$, e, em condições de campo, a aplicação de $\mathrm{N}$ aumentou a produção de matéria seca dessa gramínea (CARVALHO e SARAIVA, 1987; SKERMAN e RIVERS, 1992; SARAIVA et al. 1993; KLINK, 1994).

A flora do Parque Nacional de Brasília é apenas parcialmente conhecida. Segundo o plano de manejo, foram levantadas 631 espécies distribuídas por 370 gêneros e 98 famílias. A família mais representativa é Gramineae (95 spp), sendo destas 10 exóticas (IBAMA/ FUNATURA, 1998). Em estudo em andamento, não publicado, o primeiro autor já encontrou seis espécies de gramíneas exóticas a mais estabelecidas no Parque.

De acordo com Costa (2002), o capim-gordura está amplamente distribuído ao longo das estradas, no entanto a área total do Parque colonizada por essa gramínea não é conhecida. Estimativas preliminares indicam que essa espécie já cobre cerca de 10\% dos 30.000 hectares dessa unidade de conservação (R. R. Silva, comunicação pessoal). Devido à sua alta agressividade, essa gramínea dificulta o estabelecimento das gramíneas nativas do cerrado em áreas degradadas pela extração de cascalho laterítico (MARTINS et al., 2001).

A proteção da biodiversidade vegetal do cerrado, especialmente em áreas protegidas como Parques Nacionais e outras unidades de conservação, será mais eficiente se técnicas de controle de espécies invasoras exóticas forem desenvolvidas. O presente trabalho teve como objetivo avaliar a interferência do capimgordura (Melinis minutiflora) no processo de sucessão numa área degradada em recuperação, e seu avanço indesejável poderia ser contido pelo uso de queimada controlada. 


\section{MATERIAL E MÉTODOS}

O Parque Nacional de Brasília, criado pelo Decreto $\mathrm{n}^{\circ} 241$, de 29.11.61, possui uma área de aproximadamente 30.000 hectares e está situado entre os paralelos $15^{\circ} 34^{\prime}$ e $15^{\circ} 45^{\prime} \mathrm{S}$ e os meridianos $48^{\circ} 05^{\prime}$ e 48' 53' W, com altitudes variando entre 1.070 e 1.200 $\mathrm{m}$. Predomina na área do Parque o Latossolo Vermelho distrófico, dando suporte a um cerrado com diferentes densidades de cobertura arbórea (cerrado stricto sensu e campo cerrado). Em menor extensão ocorrem áreas com matas ciliares e campos de várzea, onde há um ligeiro incremento na oferta de nutrientes (IBAMA/FUNATURA, 1998). O clima regional, na classificação de Köppen, corresponde ao Aw, caracterizado por verões chuvosos e invernos secos (CODEPLAN, 1971).

Em estudo realizado pelo IBAMA na área do Parque, usando-se fotografias aéreas de 1975 e de 1991, foram mapeados 40 sítios com solos expostos, totalizando cerca de 180 hectares, explorados como áreas de empréstimo de terra para construção do lago de Santa Maria, que fornece água para o abastecimento de parte da cidade de Brasília e retirada de cascalho laterítico, usado na construção civil. A revegetação natural (espontânea) dessas áreas é muito baixa e em alguns casos, decorridos mais de 30 anos da desativação da exploração mineral, a superfície do solo encontra-se totalmente desnuda (LEITE et al., 1992; MARTINS, 1996).
O experimento foi implantado em uma cascalheira explorada entre 1957 e 1961. Em 1992, após três décadas de abandono, o substrato permanecia exposto, sem cobertura vegetal. Os trabalhos mecânicos de preparo do substrato foram realizados no mês de outubro de 1992. A descompactação foi realizada por meio de cinco subsoladores de $50 \mathrm{~cm}$ de comprimento, com espaçamento de $40 \mathrm{~cm}$, seguido de uma gradagem (grade de disco de 28 polegadas).

O delineamento experimental consistiu de quatro blocos de $30 \mathrm{~m}$ x $5 \mathrm{~m}$ cada, separados entre si por uma faixa de $0,5 \mathrm{~m}$. Foram demarcadas seis parcelas de $5 \mathrm{~m}$ x $5 \mathrm{~m}$, em cada bloco. Foram avaliados seis tratamentos: controle, calcário ( $5 \mathrm{t} / \mathrm{ha})$, turfa $(5 \mathrm{t} / \mathrm{ha})$, turfa $(5 \mathrm{t} / \mathrm{ha})+$ calcário $(5 \mathrm{t} / \mathrm{ha})$, torta de mamona $(5 \mathrm{t} /$ ha) e torta de mamona ( $5 \mathrm{t} / \mathrm{ha})+$ calcário $(5 \mathrm{t} / \mathrm{ha})$. A torta de mamona possuía 3,98\% de N, 0,49\% de P, 0,61\% de $\mathrm{K}, 3,93 \%$ de Ca e $2,11 \%$ de $\mathrm{Mg}$; e a turfa possuía $0,76 \%$ de $\mathrm{N}, 0,22 \%$ de $\mathrm{P}, 0,16 \%$ de $\mathrm{K}, 3,18 \%$ de $\mathrm{Ca}$ e $0,95 \%$ de $\mathrm{Mg}$.

O experimento foi implantado em dezembro de 1993 e a distribuição dos tratamentos, feita ao acaso. Os adubos e o calcário dolomítico foram incorporados manualmente, até uma profundidade de $5 \mathrm{~cm}$, com a ajuda de rastelo. Uma semana depois, foram semeados, em cada parcela, $118 \mathrm{~g}$ de um "coquetel de sementes" formado por 32 espécies de gramíneas nativas (Quadro 1). O semeio foi manual, e as sementes foram incorporadas a uma profundidade média de $2 \mathrm{~cm}$.

Quadro 1 - Espécies de gramíneas nativas do cerrado utilizadas no estudo

Table 1 - Cerrado native grass species used in the study

\begin{tabular}{ll}
\hline & Espécies \\
\hline 1 Andropogon bicornis L. & 17 Hypogynium virgatum (Desv. ex Ham.) Dandy \\
2 Andropogon leucostachyus Kunth & 18 Paspalum gardnerianum Nees \\
3 Andropogon selloanus (Hack.) Hackel & 19 Paspalum pectinatum Nees ex Trin. \\
4 Aristida gibbosa (Nees) Kunth & 20 Paspalum pilosum Lam. \\
5 Aristida recurvata Kunth & 21 Paspalum polyphyllum Nees ex Trin. \\
6 Aristida setifolia Kunth & 22 Paspalum reduncum Nees ex Steud. \\
7 Aristida torta (Nees) Kunth & 23 Paspalum splendens Hack. \\
8 Arundinella hispida (Humb. \& Bonpl. ex Willd.) Kuntze & 24 Paspalum stellatum Humb. \& Bonpl. ex Flüggé \\
9 Axonopus barbigerus (Kunth) Hitchc. & 25 Paspalum trichostomum Hack. \\
10 Axonopus canescens (Nees ex Trin.) Pilg. & 26 Setaria geniculata P. Beauv. \\
11 Ctenium cirrosum (Nees) Kunth & 27 Schizachyrium hirtiflorum Nees \\
12 Diectomis fastigiata (Sw.) P. Beauv & 28 Schizachyrium microstachyum (Desv. ex Ham.) Roseng., \\
& B.R. Arril. \& Izag. \\
13 Echinolaena inflexa (Poir.) Chase & 29 Schizachyrium tenerum Nees \\
14 Eragrostis maypurensis (Kunth) Steud. & 30 Thrasya glaziovii A.G. Burm. \\
15 Eragrostis rufescens Schrad. ex Schult. & 31 Thrasya petrosa (Trin.) Chase \\
16 Hyparrhenia bracteata (Humb. \& Bonpl. ex Willd.) Stapf & 32 Trachypogon macroglossus Trin.. \\
\hline
\end{tabular}


Quatro das oito parcelas dos dois tratamentos (torta e torta+calcário), que apresentaram os melhores índices de recobrimento do solo na avaliação realizada em maio de 1998, término da estação chuvosa, foram queimadas no final da estação seca (setembro/98). As 16 parcelas dos outros tratamentos (controle, calcário, turfa e turfa+calcário), que apresentaram baixos índices de cobertura do solo, não foram incluídas neste estudo e são denominadas, nos resultados apresentados, como outros tratamentos.

O comportamento do capim-gordura e das outras espécies foi monitorado trimestralmente entre maio de 1995 e maio de 2001. O índice de cobertura vegetal do substrato foi determinado visualmente, sendo a metodologia usada a de Barbosa et al. (1990). Em cada parcela foram marcados três transectos (distantes 1 $\mathrm{m}$ um do outro), sendo em cada transecto lançados aleatoriamente três quadrados de $1 \mathrm{~m}^{2}$, dividido em quatro quadrículas iguais e não em 16, conforme os autores citados.

\section{RESULTADOS E DISCUSSÃO}

No Quadro 2 são apresentados os resultados sobre a ocorrência das 64 espécies, semeadas e não-semeadas (recrutadas), durante o período de 1995 a 2001. Maiores informações sobre fenologia reprodutiva e características das sementes das gramíneas nativas semeadas poderão ser encontradas em Martins (1996),
Martins e Leite (1997), Carmona et al. (1998) e Carmona et al. (1999).

No ano de 1997 foi encontrado o maior número de espécies (54) na área experimental. Em decorrência da expansão do capim-gordura, o ano de 1998 foi marcado pelo início do decréscimo do número das espécies (48), chegando a 26, 25 e 20 nos três anos subseqüentes, 1999, 2000 e 2001.

O capim-gordura predomina nas pastagens das áreas montanhosas da Zona da Mata de Minas Gerais. Essas áreas caracterizam-se por elevada acidez, alta saturação de alumínio, baixa disponibilidade de fósforo e baixos níveis de bases trocáveis. A maior limitação ao crescimento do capim-gordura é a deficiência de fósforo, principalmente na fase de estabelecimento. No entanto, deficiências de potássio e de nitrogênio também limitam o crescimento inicial dessa gramínea (CARVALHO e SARAIVA, 1987).

O capim-gordura é capaz de formar uma grande biomassa em relação às outras espécies, porém essa espécie não é eficiente em explorar extensas áreas de solo em decorrência do pequeno porte de suas raízes. Um aumento na mineralização dos nutrientes resultantes de uma queimada, ou aumento de matéria orgânica, pode conduzir a uma melhoria na disponibilidade de nutrientes na superfície do solo, e nesses sítios o capim-gordura é capaz de substituir as espécies nativas.

Quadro 2 - Ocorrência no período de 1995 a 2001, das gramíneas nativas e de outras espécies não semeadas Table 2 - The occurrence of native grasses and other unseeded species during 1995-2001

\begin{tabular}{|c|c|c|c|c|c|}
\hline \multirow[t]{2}{*}{ Espécies } & \multicolumn{3}{|c|}{ Parcelas não Queimadas } & \multicolumn{2}{|c|}{ Parcelas Queimadas } \\
\hline & $\begin{array}{c}\text { Outros } \\
\text { Tratamentos }\end{array}$ & Torta & $\begin{array}{c}\text { Torta }+ \\
\text { Calcário }\end{array}$ & Torta & $\begin{array}{c}\text { Torta }+ \\
\text { Calcário } \\
\end{array}$ \\
\hline \multicolumn{6}{|c|}{ 1. GRAMÍNEAS NATIVAS SEMEADAS } \\
\hline Andropogon bicornis L. & $96,97,98$ & $\bullet$ & $\bullet$ & $\bullet$ & $\bullet$ \\
\hline Andropogon leucostachyus Kunth & $95,96,97$ & $\bullet$ & $\bullet$ & $\bullet$ & $\bullet$ \\
\hline Aristida gibbosa (Nees) Kunth & $\begin{array}{c}95,96,97 \\
98\end{array}$ & $\begin{array}{l}96,97,98, \\
98,00,01\end{array}$ & $96,97,00,01$ & $99,00,01$ & $\bullet$ \\
\hline Aristida recurvata Kunth & 96 & $\bullet$ & $\bullet$ & $\bullet$ & $\bullet$ \\
\hline Aristida setifolia Kunth & $95,96,97,98$ & $96,97,98$ & $98,00,01$ & $99,00,01$ & $\bullet$ \\
\hline Aristida torta (Nees) Kunth & $\bullet$ & $\bullet$ & 97 & $\bullet$ & - \\
\hline Arundinella hispida (Humb. \& Bonpl. ex Willd.) Kuntze & $\bullet$ & 99 & $98,96,99$ & $\bullet$ & $\bullet$ \\
\hline Axonopus barbigerus (Kunth) Hitchc. & $\begin{array}{c}96,97,98 \\
96,97,98,99\end{array}$ & $\begin{array}{l}00,01,96, \\
97,98,99\end{array}$ & 00,01 & 00,01 & $\bullet$ \\
\hline
\end{tabular}


Quadro 2, cont.

Table 2, cont.

Espécies

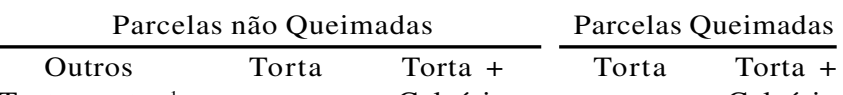

Tratamentos ${ }^{1}$ Calcário Calcário

Axonopus canescens (Nees ex Trin.) Pilg.

Ctenium cirrosum (Nees) Kunth

Diectomis fastigiata (Sw.) P. Beauv.

Echinolaena inflexa (Poir.) Chase

Eragrostis maypurensis (Kunth) Steud.

Hyparrhenia bracteata (Humb. \& Bonpl. ex Willd.) Stapf

Paspalum gardnerianum Nees

Paspalum pectinatum Nees ex Trin.

Paspalum pilosum Lam.

Paspalum polyphyllum Nees ex Trin.

97,98

97,98
97,98

$95,96,97,98$

$95,96,97$

97,98

95,98

97,98

97,98

Paspalum reduncum Nees ex Steud.

$95,96,97,98$

96,97,98,99

$96,97,98$

$95,96,97$

95,96,97,98

95,96,97,98

$95,96,97,98$

$96,97,98,99$

Paspalum splendens Hack.

$95,96,97,98$

$95,96,97,98$,

$99,00,01$

Paspalum stellatum Humb. \& Bonpl. ex Flüggé

$95,96,97,98$

$96,97,98$,

Schizachyrium hirtiflorum Nees

$95,96,97,98$

$99,00,01$
$95,96,97$
$96,97,99,00$

Schizachyrium microstachyum (Desv. ex Ham.)

$$
96,97,98
$$

96,97

Roseng., B.R. Arrill. \& Izag.

Setaria geniculata P. Beauv.

$95,96,97,98$

$95,96,97$ $98,99,00$

$95,96,97,98$

$95,97,98$

97

$98,99,00, \quad 98,99,00,01$

$\bullet$

$01,95,96,97$,

$$
95,96,97, \quad 98,99,00,01
$$

$97,98,99, \quad 00,01$

$97,98 \quad 96,97,98$

$95,96,97,98 \quad 97,98,99,00$

$97,98,99, \quad 00,01$

00

$00,01 \quad 99,00,01 \quad 99,00,01$

$96,97,98$,

$97,98,99$,

00,01

99

$8,99,00,01$

$96,99,01 \quad 99,00,001$

$99,00,01$

Thrasya glaziovii A.G. Burm.

•

-

(n)
$96,97,99$
00,0

$96,97,98$, $99,00,01$

$96,97,99$

$96,97,99, \quad 99,00,01$

2. COLONIZADORAS ESPONTÂNEAS

2.1. GRAMÍNEAS NATIVAS

Digitaria $s p$

Gymnopogon spicatus Spreng.) Kuntze

Panicum campestre Nees ex Trin.

Paspalum plicatulum Michx.

$\begin{array}{ccccc}95 & \bullet & \bullet & \bullet & \bullet \\ 96,97 & & & & \\ 95,96 & \bullet & 97 & \bullet & \bullet \\ \bullet & \bullet & 95,96 & \bullet & \bullet\end{array}$

2.2. GRAMÍNEAS EXÓTICAS

Andropogon gayanus Kunth

$\bullet$

$\bullet$

97,98

$99,00,01$

98,99,

$95,96,97,98$

$95,96,97$

$95,96,97$

$99,00,01$

$99,00,01$

Melinis minutiflora P. Beauv. $98,99,00,0198,99,00,01$

Pennisetum $s p$

Rhynchelytrum repens (Willd.) C.E. Hubb.

$96,97,98$
$95,96,97,98$

$96 \quad 96,97$

95,96,97, 95,96,97,

$98,99,00,0198,99,00,01$

99,00,01 $99,00,01$

.

2.3. CYPERACEAE

Bulbostylis sp

Cyperus rotundus $\mathrm{L}$.

95,96,97,98

$95,96,97,98$

95,96

$95,96,97$

Cyperus $s p$

96

96

\begin{tabular}{rrr}
$\bullet$ & $\bullet$ & $\bullet$ \\
$\bullet$ & $\bullet$ & $\bullet$ \\
$\bullet$ & $\bullet$ & $\bullet$ \\
\hline
\end{tabular}

Continua... Continued...

R. Árvore, Viçosa-MG, v.28, n.5, p.739-747, 2004 
Quadro 2, cont

Table 2, cont.

Espécies

\begin{tabular}{|c|c|c|c|c|}
\hline \multicolumn{3}{|c|}{ Parcelas não Queimadas } & \multicolumn{2}{|c|}{ Parcelas Queimadas } \\
\hline $\begin{array}{c}\text { Outros } \\
\text { Tratamentos }\end{array}$ & & $\begin{array}{c}\text { Torta }+ \\
\text { Calcário }\end{array}$ & Torta & $\begin{array}{c}\text { Torta }+ \\
\text { Calcário }\end{array}$ \\
\hline
\end{tabular}

2.4. HERBÁCEA/ARBUSTIVA

Achyrocline alata (Kunth) DC.

\begin{tabular}{|c|c|c|c|c|}
\hline $95,96,97,98$ & $\begin{array}{c}95,96,97 \\
98,99\end{array}$ & $\begin{array}{c}95,96,97 \\
98,99\end{array}$ & 00,01 & 99,00 \\
\hline $95,96,97,98$ & $95,96,97,98$ & $\begin{array}{c}95,96,97 \\
98,99\end{array}$ & - & 99,00 \\
\hline 97 & - & $\bullet$ & - & - \\
\hline $96,97,98$ & 97,98 & $96,97,98$ & 00 & - \\
\hline $95,96,97$ & 95,96 & 97 & & $\bullet$ \\
\hline $95,96,97,98$ & $\begin{array}{c}95,96,97, \\
98,99,00,01\end{array}$ & $\begin{array}{l}96,97,98, \\
99,00,01\end{array}$ & $99,00,01$ & $99,00,01$ \\
\hline $96,97,98$ & 96,98 & 98 & 99,00 & 99,00 \\
\hline$\bullet$ & 95 & 98 & $\bullet$ & $\bullet$ \\
\hline - & - & 98,99 & 00 & - \\
\hline 97 & 97 & 95,97 & 97 & - \\
\hline - & - & $95,96,97,98$ & - & - \\
\hline - & - & 98 & - & - \\
\hline 97 & - & 97 & - & - \\
\hline$\bullet$ & $\bullet$ & 95,96 & - & - \\
\hline $95,96,97$ & 90,97 & $95,96,97$ & - & - \\
\hline 96,97 & $\bullet$ & 96,97 & - & $\bullet$ \\
\hline \multirow[t]{2}{*}{$96,97,98$} & $\bullet$ & 96,97,98, & $\bullet$ & 00,01 \\
\hline & & $99,00,01$ & & \\
\hline$\bullet$ & $96,97,98$ & $\bullet$ & $\bullet$ & $99,00,01$ \\
\hline $96,97,98$ & 97 & 97,98 & $\bullet$ & $\bullet$ \\
\hline 97,98 & - & $\bullet$ & - & - \\
\hline 96 & - & - & - & - \\
\hline 97,98 & 00 & $\bullet$ & - & - \\
\hline$\bullet$ & - & 97,98 & - & - \\
\hline 96,97 & 00 & $\bullet$ & - & - \\
\hline $95,96,97,98$ & 98 & $\begin{array}{c}95,96,97, \\
98,99,00,01\end{array}$ & - & $99,00,01$ \\
\hline $95,96,97,98$ & $\begin{array}{c}95,96,97, \\
98,99,00,01\end{array}$ & $96,97,98,99$ & $99,00,01$ & $99,00,01$ \\
\hline $95,96,97,98$ & $\begin{array}{c}95,96,97 \\
98,99\end{array}$ & $\begin{array}{c}96,97,98 \\
98,99\end{array}$ & 99 & $99,00,01$ \\
\hline $96,97,98$ & $\begin{array}{l}96,97,98, \\
99,00,01\end{array}$ & $96,97,98$ & - & 98 \\
\hline - & $\begin{array}{c}96,97,98 \\
99,00\end{array}$ & 00 & 00,01 & - \\
\hline
\end{tabular}

Mimosa gracilis Benth.

Polygala sp

Porophyllum ellipticum Cass.

Schwenkia americana $\mathrm{L}$.

Scoparia $s p$

Sida sp

Solanum americanum Mill.

Solanum granuloso-leprosum Dunal

Solanum lycocarpum A. St.-Hil.

Trichogonia salviifolia Gardner

Vernonia aurea Mart. ex DC.

Vernonia fruticosa (L.) Sw.

Vernonia $s p$

1 - O tratamento outros representa todas as espécies encontradas nos tratamentos-controle, calcário, turfa e turfa+calcário (MARTINS, 1996).

Nesses casos, o solo pode ter características diferentes do ótimo fisiológico para os processos de colonização das espécies nativas pré-existentes do local (PIANKA, 1983, citado por DURINGAN et al., 1998). Devido à grande capacidade de expansão vegetativa, resultante da total conversão de nutrientes em biomassa alocado para as folhas (BARUCH et al., 1985), o capim-gordura pode formar uma densa camada de folhas, promovendo uma grande vantagem competitiva sobre as demais espécies do estrato rasteiro, sendo responsável pela diminuição da biodiversidade na área estudada.

Oito meses após a realização da queimada, verificou-se nos dois tratamentos, torta e torta+calcário, uma diminuição no índice de cobertura do solo, passando de $67 \%$ e $85 \%$ em maio de 1998 antes da queimada, para $29 \%$ e $42 \%$, respectivamente. Nos anos subseqüentes, o índice de cobertura voltou a crescer, al- 
cançando, na avaliação realizada em maio de 2001, uma situação próxima da cobertura verificada antes da realização da queimada (Figura 1a).

A contribuição do capim-gordura para o índice total de cobertura do solo não apresentou padrão definido. Oito meses após a realização da queimada, a contribuição do capim-gordura, que era de 15\% (maio/98), passou para $13 \%$ (maio/99) no tratamento torta e, no tratamento torta+calcário, passou de $16 \%$ para $25 \%$. Já nas parcelas queimadas, a contribuição do capim gordura caiu de $24 \%$ (maio/98) para $12 \%$ (maio/99) no tratamento torta e, no tratamento torta+calcário, passou de $16 \%$ para $22 \%$ (Figura $1 \mathrm{~b}$ )

Os valores máximos de cobertura do solo e da contribuição do capim-gordura foram registrados em maio de 2000 , na segunda avaliação. No ano seguinte (maio/2001), nas parcelas queimadas e não queimadas, verificou-se um decréscimo da cobertura do solo, a qual se aproximou do índice verificado em maio de 1998 (antes da queimada). Em maio de 1999, nas parcelas queimadas a redução da cobertura do solo foi de cerca de 50\%. Em maio de 2001, nas parcelas queimadas a contribuição do capim-gordura para a cobertura total praticamente dobrou, em comparação com o índice verificado um ano após a queimada (maio/ 1999), o que demonstra que o fogo não foi eficaz no controle do capim-gordura.

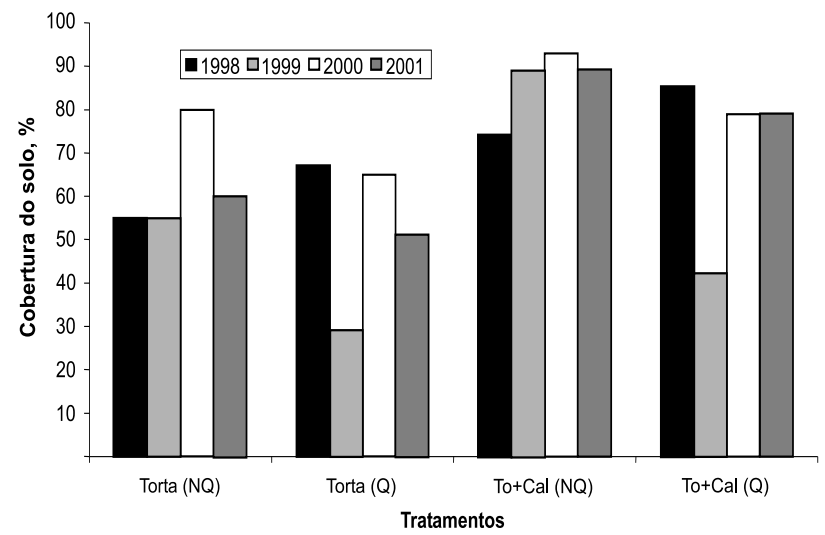

\section{CONCLUSÕES}

O capim-gordura, pela sua alta agressividade, dificulta o estabelecimento de gramíneas nativas do cerrado em áreas degradadas pela extração de cascalho laterítico.

A espécie Melinis minutiflora (capim-gordura) não foi semeada, mas se estabeleceu na maioria dos tratamentos do experimento implantado em 1993, para estudar a possibilidade de usar gramíneas nativas do cerrado para recuperar áreas degradadas pela extração de cascalho, localizadas no Parque Nacional de Brasília.

A revegetação de áreas degradadas pela extração de cascalho laterítico, pelo emprego da técnica do "coquetel de sementes" formado por gramíneas nativas do cerrado, requer o desenvolvimento e aplicação de práticas para controlar o estabelecimento do capim-gordura.

A queimada controlada, realizada no final do período seco da região, não se mostrou eficaz no controle do capim-gordura nas parcelas experimentais. No período avaliado, maio de 1998 a maio de 2001, a contribuição do capim-gordura para a cobertura do solo aumentou em detrimento das 32 gramíneas nativas do cerrado, usadas na formação do "coquetel de sementes".

Figura 1 - Cobertura do solo (A) e contribuição do capim-gordura (B) para a cobertura do solo $(\mathrm{Q}=$ queimada, $N Q=$ não-

queimada e $\mathrm{To}+\mathrm{Ca}=$ torta de mamona + calcário)
Figure 1 - Vegetative cover $(A)$ and the contribution of Melinis minutiflora $(B)$ to the vegetative cover $(Q=$ burned, $N Q$

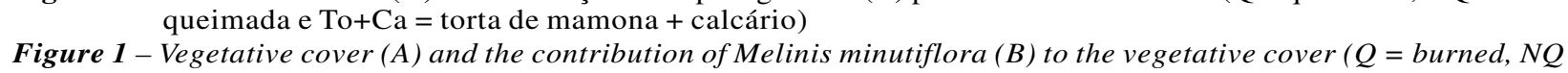
= unburned, $T o+C a=$ castor bean cake + limestone)

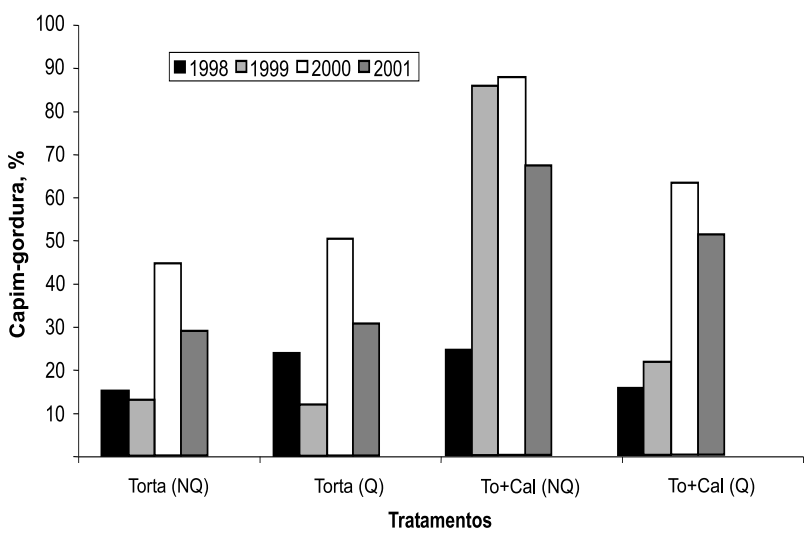




\section{AGRADECIMENTO}

Os autores agradecem ao IBAMA/Parque Nacional de Brasília pelo importante apoio prestado à realização dos trabalhos de campo; e aos consultores anônimos pelas sugestões.

\section{REFERÊNCIAS BIBLIOGRÁFICAS}

BARBOSA, J.M. et al. Gramíneas pioneiras ocorrentes em áreas degradadas da Serra do Mar: produção de sementes, germinação e capacidade de ocupação das espécies. Ecossistemas, v. 15: p. $65-73,1990$.

BARUCH, Z.; LUDLOW, M.M.; DAVIS, R. Photosynthetic responses of native and introduced $\mathrm{C}_{4}$ grasses from Venezuela savannas. Oecologia. v.67, p.388-393, 1985.

CARMONA, R.; MARTINS, C.R.; FÁVERO, A.P. Fatores que afetam a germinação de sementes de gramíneas nativas do cerrado. Revista Brasileira de Sementes, v.20, n.1, p.16-22, 1998.

CARMONA, R.; MARTINS, C.R.; FÁVERO, A.P. Características de sementes de gramíneas nativas do cerrado. Pesquisa Agropecuária

Brasileira, v.34, n.6, p.1067-1074, 1999.

CARVALHO, M.M.; SARAIVA, O.F. Resposta do capim-gordura (Melinis minutiflora Beauv.) a aplicações de nitrogênio, em regime de cortes. Revista da Sociedade Brasileira de Zootecnia. v.16, n.5, p.442-454, 1987.

Companhia de Desenvolvimento do Planalto. CODEPLAN. Diagnóstico do espaço natural do Distrito Federal. Brasília: 1971. 300 p.

CostA, E.M.M. Liquens e capins invasores como indicadores do estado de conservação das fisionomias de cerrado do Parque Nacional de Brasília. 2002. 116f. Tese (Doutorado em Botânica) - Universidade Federal de Brasília, Brasília, 2002.

D’ANTONIO, C.M.; VITOUSESK, P.M. Biological invasions by exotic grasses, the grass/fire cycle, and global change. Annual Review Ecology Systens. v.23. p.63-87, 1992.

R. Árvore, Viçosa-MG, v.28, n.5, p.739-747, 2004
DURIGAN, G. et al. Indução do processo de regeneração da vegetação de cerrado em área de pastagem, Assis, SP. Acta Botânica Brasileira. v.12, n.3, p.421-429, 1988.

FILGUEIRAS, T.S. A floristic analysis of the gramineae of Brasil's Distrito Federal and a list of the species occurring in the area. Edinburgh Journal of Botany, v.48, n.1, p.73-80, 1991.

FREITAS, G.K. Invasão biológica pelo capim-gordura (Melinis minutiflora Beauv) em um fragmento de cerrado (A.R.I.E Cerrado Pé-de-Gigante, Santa Rita do Passa Quatro,SP). 1999. $152 \mathrm{f}$. Dissertação (Mestrado em Biociências) - Instituto de Biociências da Universidade de São Paulo, São Paulo, 1999.

IB AMA/FUnAturA. Plano de Manejo do Parque Nacional de Brasília. Brasília, 1998. v.1.

KLINK, C.A. Effects of clipping on size and tillering of native and african grasses of the brazilian savannas (the cerrado). Oikos, 70: 365376, 1994.

LEITE, L.L.; MARTINS C.R.; HARIDASAN, M. Propriedades físico-hídricas do solo de uma cascalheira e de áreas adjacentes com vegetação nativa de campo sujo e cerrado no Parque Nacional de Brasília. In: SIMPÓSIO NACIONAL SOBRE RECUPERAÇÃO DE ÁREAS

DEGRADADAS, 1., 1992, Curitiba. Anais... Curitiba: UFPR/Fundação de Pesquisas Florestais do Paraná, 1992. p.392-399.

MARTINS, C.R. Revegetação com gramíneas nativas de uma área degradada no Parque Nacional de Brasília, DF, Brasil. 1996, 10f. Dissertação (Mestrado em Ciências Florestais) - Universidade de Brasília, Brasília, 1996.

MARTINS, C.R.; LEITE, L.L. Fenologia reprodutiva de gramíneas colonizadoras de áreas degradadas no Parque Nacional de Brasília. In: SIMPÓSIO NACIONAL RECUPERAÇÃO DE ÁREAS DEGRADADAS, 3., 1997. Viçosa. Anais... Ouro Preto/MG: Universidade Federal de Viçosa, 1997. p. 317-323. 
MARTINS, C.R.; LEITE, L.L.; HARIDASAN, M. Recuperação de uma área degradada pela mineração de cascalho com uso de gramíneas nativas. Revista Árvore, v.25, n.2, p. 157-166, 2001.

MENDONÇA, R.C. et al. Flora vascular do Cerrado. In: SANO, S.M.; ALMEIDA, S.P. Cerrado: ambiente e flora. Planaltina: EMBRAPA-CPAC, 1998. 556 p.

MOROSINI, I.B.A.; KLINK, C.A. Interferência do capim-gordura (Melinis minutiflora Beauv.) no desenvolvimento de plântulas de embaúba (Cecropia pachystachya). In: LEITE, L.L; SAITO C.H. (Org). Contribuição ao conhecimento ecológico do cerrado. In: CONGRESSO DE ECOLOGIA DO BRASIL, 3., 1997, Brasília. Trabalhos selecionados ... Brasília: Universidade de Brasília, Brasília, 1997.

PIVELLO, V.R. et al. Abundance and distribution of native and alien grasses in a "cerrado" (Brazilian savannas) Biological Reserve.

Biotropica, v.31, n.1, p. 72-82, 1999.
SARAIVA, O.D.; CARVALHO, M.M.;OLIVEIRA, F.T.T. Nutrientes limitantes ao crescimento de capim-gordura em um latossolo vermelho-amarelo álico. Pesquisa Agropecuária Brasileira. Brasília, v.284, n.8, p.963-968, 1993.

SILVA, J.S.O. Acúmulo de biomassa aérea e concentração de nutrientes em Melinis minutiflora e gramíneas nativas do Cerrado. 2003. 48f. Dissertação (Mestrado em Biociências) - Universidade de Brasília,

Brasília, 2003.

SKERMAN, P.J.; RIVERS, F.R. Gramineas tropicales. Roma: FAO, 1992. 849p. (Coleción FAO: Producción y protección vegetal, 23), 1992. 849 p.

UNESCO - Vegetação no Distrito Federal - tempo e espaço. Brasília; 2000. 74 p. 